\title{
Power indices of influence games and new centrality measures for agent societies and social networks ${ }^{\star}$
}

\author{
Xavier Molinero ${ }^{1}$, Fabián Riquelme ${ }^{2}$, and Maria Serna ${ }^{2}$ \\ 1 Dept. of Applied Mathematics III. UPC, Manresa, Spain. \\ E-mail: xavier.molinero@upc.edu \\ 2 Dept. de Llenguatges i Sistemes Informàtics. UPC, Barcelona, Spain. \\ E-mail: $\{$ farisori,mjserna $\} @ l s i . u p c . e d u$
}

\begin{abstract}
We propose as centrality measures for social networks two classical power indices, Banzhaf and Shapley-Shubik, and two new measures, effort and satisfaction, related to the spread of influence process that emerge from the subjacent influence game. We perform a comparison of these measures with three well known centrality measures, degree, closeness and betweenness, applied to three simple social networks.
\end{abstract}

Keywords: Social Network, Centrality, Power index, Influence game, Simple game

\section{Introduction and preliminaries}

We propose to study networked societies, social networks or agent societies, from the social networking point of view. Social network analysis is a multidisciplinary field related to sociology, computer science and mathematics, among other topics. One of the most studied concepts is centrality, that measures how structurally important is an actor within a social network $[4,15,8,12]$. Here we consider seven centrality measures: Banzhaf and Shapley-Shubik power indices through the use of influence games [10]; two new measures, the effort and the satisfaction; and the classic ones [8], degree, closeness and betweenness. We perform an experimental comparison on three simple real social networks, monkeys' interaction $[3,8]$, dining-table partners [11,2], and student Government discussion [6, 2].

A social network is a directed edge-labeled graph $(G, w)$, where $G=(V, E)$ is a graph without loops, $V$ is the set of nodes representing individuals, actors, players, etc., $E$ is the set of edges representing interpersonal ties between actors, and $w: E \rightarrow \mathbb{R}$ is a weight function which assigns a weight to every edge, representing the strength of each interpersonal tie. An actor $i \in V$ has influence over another $j \in V$ if and only if $(i, j) \in E$.

\footnotetext{
* This work was partially supported by 2009SGR1137 (ALBCOM). X. Molinero, F. Riquelme and M. Serna are also partially funded by grants MTM 2012-34426, BecasChile (CONICYT), and TIN2007-66523 (FORMALISM), respectively.
} 
Now we consider three of the most well-known (normalized) centrality measures [8], which study the relevance of a node inside a network [15]. We use the notation $\mathrm{deg}^{-}(i)=|\{j \in V \mid(j, i) \in E\}|$ and $\mathrm{deg}^{+}(i)=|\{j \in V \mid(i, j) \in E\}|$.

Degree centrality $\left(C_{D}\right)$ : measures the average indegree or outdegree of each actor, $C_{D}^{-}(i)=\operatorname{deg}^{-}(i) /(n-1)$, or $C_{D}^{+}(i)=\operatorname{deg}^{+}(i) /(n-1)$. For undirected networks, $\operatorname{deg}(i)=\operatorname{deg}^{-}(i)=\operatorname{deg}^{+}(i)$, so we set $C_{D}=C_{D}^{-}=C_{D}^{+}$.

Closeness centrality $\left(C_{C}\right)$ : It is based on the inverse of the sum of the shortest distances from $i$ to the other actors. Let $D$ be the usual distance matrix of the network in which, if there is no path from $i$ to $j$, we set $(D)_{i j}=n$. We define $C_{C}(i)=(n-1) / \sum_{i \neq j}(D)_{i j}$.

Betweenness centrality $\left(C_{B}\right)$ : Let $b_{j k}$ the number of shortest paths from the node $j$ until $k$, and $b_{j i k}$ the number of these shortest paths that pass through $i$. If there is no path from $j$ to $k$, we assume that $b_{j i k} / b_{j k}=0$. We define $C_{B}(i)=\sum_{j \neq k} \frac{b_{j i k}}{b_{j k}} /((n-1)(n-2))$.

Notation related to simple and influence games comes from $[13,10]$. An influence graph is a tuple $(G, w, f)$ where $(G, w)$ is asocial network and $f: V \rightarrow \mathbb{N}$ a labeling function that quantifies how influenciable each actor is.

Given an influence graph $(G, w, f)$ and an initial activation set $X \subseteq V$, the spread of influence [7], in the linear threshold model, is denoted by $F(X)$, where $F(X) \subseteq V$ is formed by the actors activated through an iterative process in which initially only the nodes in $X$ are activated. Let $F^{t}(X)$ be the set of nodes activated at some iteration $t$, then at the next $t+1$ iteration a node $i \in V$ will be activated iff $\sum_{j \in F^{t}(X)} w((j, i)) \geq f(i)$. The process stops when no additional activation occurs.

A simple game is a tuple $(N, \mathcal{W})$ where $N$ is a finite set of players and $\mathcal{W}$ is a monotonic family of subsets of $N$ formed by the winning coalitions, such that if $X \in \mathcal{W}$ and $X \subseteq Z$, then $Z \in \mathcal{W}$. An influence game is a simple game defined by a tuple $(G, w, f, q)$ where $(G, w, f)$ is an influence graph and $q$ is a quota $0 \leq q \leq|V|+1$. $X \subseteq V$ is a winning coalition iff $|F(X)| \geq q$, otherwise $X$ is a losing coalition. Note that every simple game is an influence game [10]. From now on, we assume $N=V$ and $n=|N|=|V|$.

\section{Power indices and new centrality measures}

A power index is a measure of the relevance of the players in a game $[1,5]$. We consider the two main power indices of a given simple game $(N, \mathcal{W})$. The Banzhaf index $\mathrm{Bz}(i)=\left|C_{i}\right| / \sum_{i \in N}\left|C_{i}\right|$ and the Shapley-Shubik index $\operatorname{SS}(i)=$ $\left(\sum_{S \in C_{i}}(|S|-1) !(n-|S|) !\right) / n$ !, where $C_{i}=\{S \in \mathcal{W} \mid S \backslash\{i\} \notin \mathcal{W}\}$.

Power indices, in influence games, can be considered centrality measures because an actor is more central in a network while more necessary is for generating of winning coalitions. Moreover, influence games also provide other new criteria to determine measures of centrality. Let $f(S)=\sum_{i \in S} f(i)$, for a coalition $S \subseteq N$. For an influence game $(G, w, f, q), \operatorname{Effort}(i)=\min \{f(S)|| F(S \cup\{i\}) \mid \geq q\}$, the (minimum) effort required by the network to choose a winning coalition that 

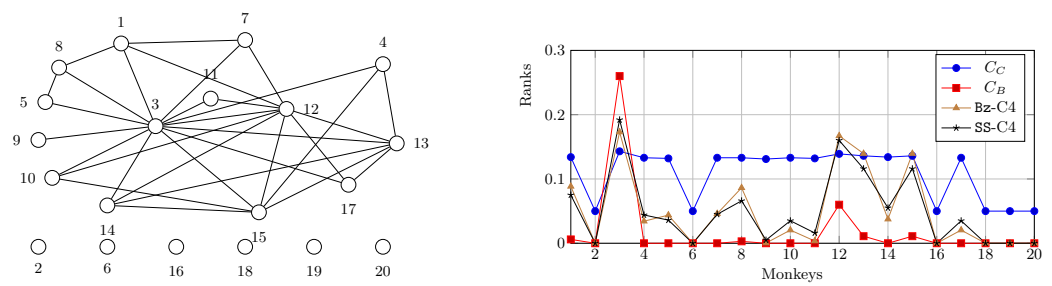

Fig. 1. Monkeys' interaction network and comparisons among Bz-C4, SS-C4, $C_{C}, C_{B}$.

contains a required actor. While greater is the required effort for a node, this node should be less central. Therefore, the effort centrality measure is the effort required to make the social network follows the opinion of an actor, i.e., $C_{E}(i)=(f(N)-\operatorname{Effort}(i)) / f(N)$.

The second new measure is the satisfaction centrality measure, based on the satisfaction score [14], representing the level of satisfaction of each actor applied to an influence game $(G, w, f, q)$, i.e., $C_{S}(i)=\left(\left|\mathcal{W}_{i}\right|+\left|\mathcal{L}_{-i}\right|\right) / 2^{n}$, where $\mathcal{W}_{i}=$ $\{X \subseteq V(G)|i \in X| F,(X) \mid \geq q\}$ and $\mathcal{L}_{-i}=\{X \subseteq V(G)|i \notin X| F,(X) \mid<q\}$.

\section{Cases of study}

We consider three simple real social networks to compare the new centrality measures Bz, SS, $C_{E}$ and $C_{S}$, with some traditional ones, $C_{D}, C_{C}$ and $C_{B}$. In each comparative table the three more central values will be highlighted in bold. We used enough significant digits to distinguish all the different values.

Monkeys' interaction. This is a network representing the real interactions amongst a group of 20 monkeys observed during three months next to a river provided in [3]. It is represented by an undirected graph with an edge $\{i, j\}$ whenever monkeys $i$ and $j$ were witnessed together in the river. See Figure 1, on the left.

In order to analyze this network $((V, E), w)$ we assume, as usual, that every undirected edge $\{i, j\}$ with $i, j \in V$ represents in fact two $\operatorname{arcs}(i, j)$ and $(j, i)$ of $E$, and the weight function is defined by $w(e)=1$, for all $e \in E$. In our context, this means that a monkey can influence and be influenced by other monkey if and only if they have interacted. To define an influence game we have to set the quota and define the labeling function. We select $q=14$, which corresponds to the maximum spread of influence which can be obtained from a monkey. We consider four labeling functions representing different influence requirements. For every node $i \in V,(\mathrm{C} 1)$ minimum, $f(i)=1 ;(\mathrm{C} 2)$ average, $f(i)=\lceil\operatorname{deg}(i) / 2\rceil$; (C3) majority, $f(i)=\lfloor\operatorname{deg}(i) / 2\rfloor+1$; and $(\mathrm{C} 4)$ maximum, $f(i)=\operatorname{deg}(i)$.

The Bz, SS, $C_{E}$ and $C_{S}$ measures have been computed for all these cases (Table 1). Note that only isolated nodes for $\mathrm{Bz}, \mathrm{SS}$ and $C_{E}$, as well as the last column of $C_{E}$ assume a score exactly equal to zero. For (C1), the new measures are not good representatives. As the spread of influence is fluid, i.e., actors 


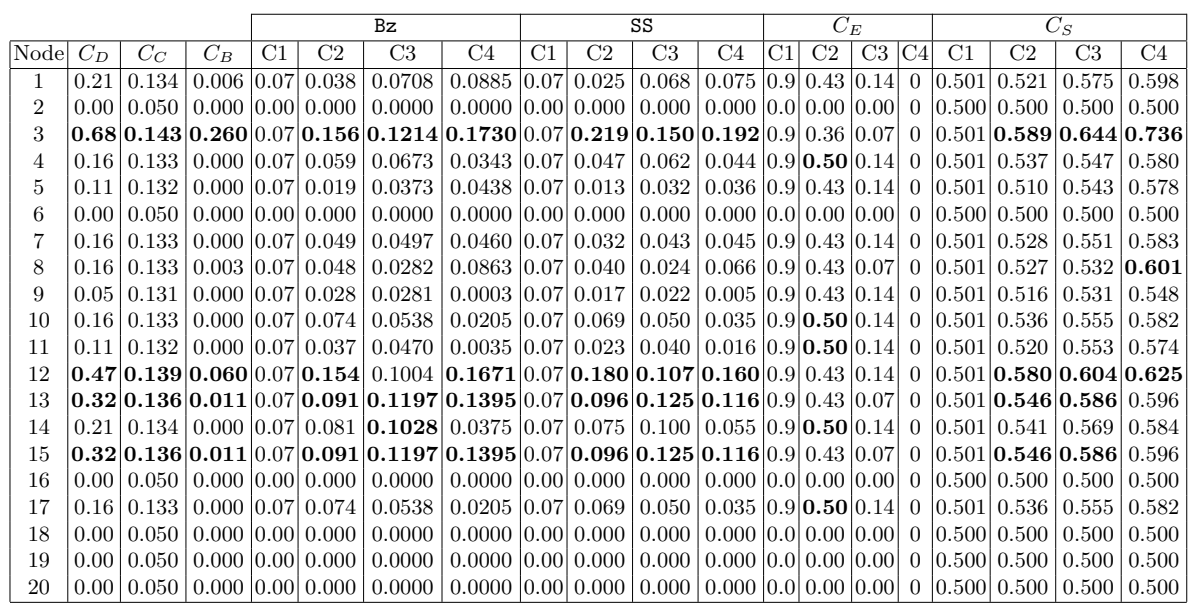

Table 1. Comparison for the Monkeys' interaction network for $q=14$.

do not require too many restrictions to form winning coalitions, then all the non-isolated nodes have the same value. However, for the other cases in which differences between influence are relevant, only the pair of monkeys $(10,17)$ and $(13,15)$ assume the same value for $\mathrm{Bz}, \mathrm{SS}$ and $C_{S}$, allowing a more relevant classification.

Dining-table partners. This network represents the companion preferences of 26 girls living in one cottage at a New York state training school [11,2]. See Figure 2, on left. Each girl was asked about who prefers as dining-table partner in first and second place. Thus, each girl is represented by a node, and there is a directed edge $(i, j)$ per each girl $i$ preferring girl $j$ as dining-table partner. Every node has an outdegree equal to 2 : edges with weight 1 denote the first option of the girl, and edges with weight 2 denote her second option.

We could assume that a girl has some ability to influence another one which has chosen her as a partner. Figure 2 (on right) shows the corresponding network of this influence game, reversing each arc $(i, j)$ by $(j, i)$, so that a node points to another when the first one has some influence over the second one. Further, the weights of the edges must be exchanged, so that an original edge $(i, j)$ with weight 1 now becomes in an edge $(j, i)$ with weight 2 , and viceversa. Because a girl has more influence over another one if that other has chosen her in the first place rather than in the second place. Of course, now every node has an indegree equal to 2: one edge with weight 1 and the other with weight 2 . We consider a quota $q=14$, so that a coalition is winning if and only if it achieves to convince (through its spread of influence) most of the girls absolute majority. For every node $i \in V$, we consider the following labeling functions:

(C1) minimum, $f(i)=1$; (C2) average, $f(i)=2$; and (C3) maximum, $f(i)=3$. Unlike in the previous network, here there are no isolated nodes, but we can 

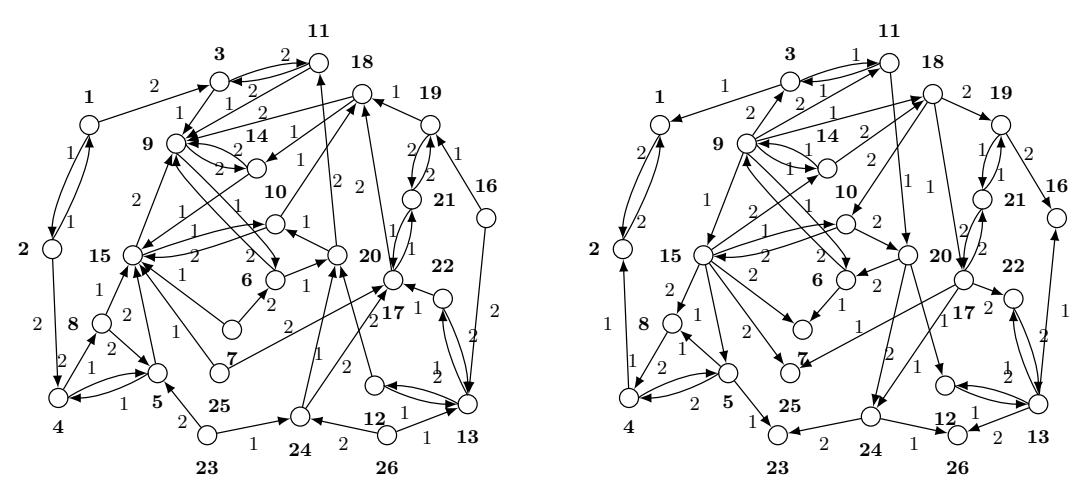

Fig. 2. Network for dining-table partners and the associated influence graph.

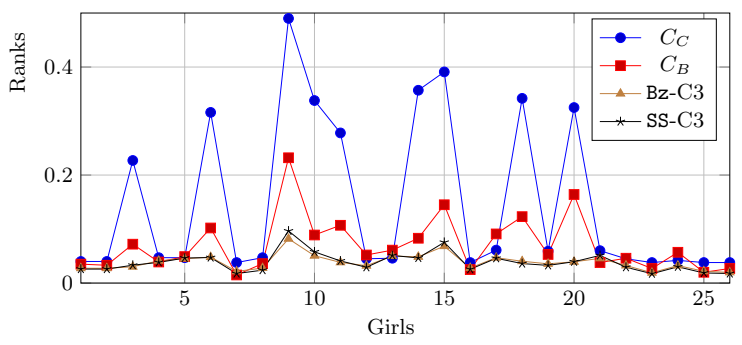

Fig. 3. Comparative between Bz-C3, SS-C3, $C_{C}$ and $C_{B}$ for Dining-table partners.

still obtaining scores for $\mathrm{Bz}$ and SS equal to zero. See the columns of Bz-C1 and SS-C1 on Table 2.

Indegree centrality $C_{D}^{-}$does not provide any relevant information, because the indegree for each node is always 2 (see Table 2 and Figure 3 ). Similarly as it succeded in the previous network, Bz-C1, SS-C1, $C_{E^{-}} \mathrm{C} 1$ and $C_{S^{-}} \mathrm{C} 1$ have several nodes with the same rank, but while the required influence to convincement increases, the values of the measures are more diverse for the power indices and satisfaction centrality. Measures Bz-C2, SS-C2 and $C_{S}-\mathrm{C} 2$, as well as $C_{D}^{+}$and $C_{C}$, have only some values that are repeated, but measures Bz-C3, SS-C3 and $C_{S}$-C3 have the same values only for girls 1 and 2 . These girls are equivalent in this sense for all the other measures except by $C_{B}$, in which, however, together with $C_{E}$, girls 23 and 26 have the same centrality.

Girl 15 has a high centrality in all measures, as well as girl 9, except in $C_{E}-\mathrm{C} 2$, as well as in Bz-C2 and SS-C2, where is far less central. Girl 13 is fairly central exclusively in $C_{D}^{+}$, because despite of its high outdegree, only exist paths from this node to another four, which is a severe restriction for all other measures. 


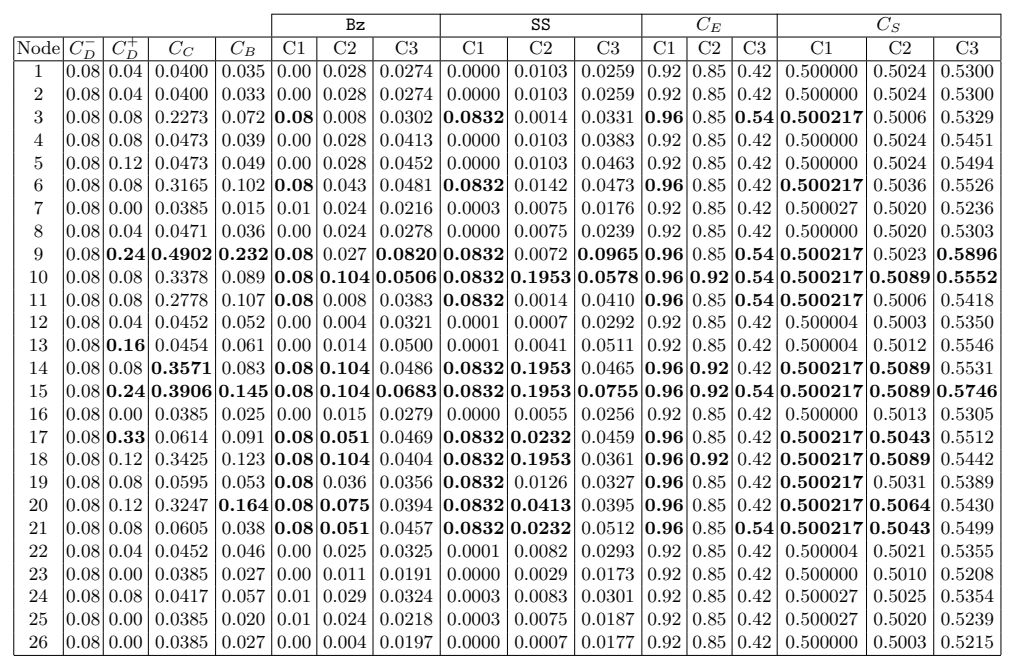

Table 2. Comparison values for the Dining-table partners network for $q=14$.

Student Government discussion. The last case of study starts with the social network illustrated in Figure 4. This network represents the communication interactions among different members of the Student Government at the University of Ljubljana in Slovenia. Data were collected through personal interviews in 1992 and published by [6], being used later by [2] .

Every directed edge is a communication interaction and all of them have the same weight equal to 1. Each node is a member of the Student Government, and unlike the previous cases, here nodes are labeled beforehand: There are three advisors labeled 1, seven ministers labeled 2, and one prime minister labeled 3.

We modified slightly this network to obtain the influence graph of Figure 4. We assume that every communication interaction is an attempt to influence another student. Thus, the capacity to influence depends on the student's position. For instance, the advise of a prime minister does not have the same effectiveness - marked with weight 3- than the advise of an advisor - marked with weight 1. Furthermore, as the labels of the nodes should represent the difficulty of each student $i \in N$ to be influenced, according to their position in the Student Government, then they have been changed by the following values: $f(i)=1$, if $i$ is an advisor; $f(i)=\left\lceil\mathrm{deg}^{-}(i) / 2\right\rceil$, if $i$ is a minister; and $f(i)=\operatorname{deg}^{-}(i)$, if $i$ is the primer minister. We consider a majority influence required to win, setting $q=6$ (see Table 3 and Figure 4 ).

Traditional measures provide different rankings. In fact, none of the most central nodes measured with $C_{C}$ and $C_{B}$ coincide, and while the most central node for $C_{C}$ is the advisor 10, this is the less central according to $C_{B}$. Moreover, the ministers 3 and 1 are very central for $C_{C}$ but with $C_{B}$ are at the bottom of the ranking. This is because nodes 1,3 and 10 have a high accessibility to all other nodes, but however, they are not good intermediaries for connecting 

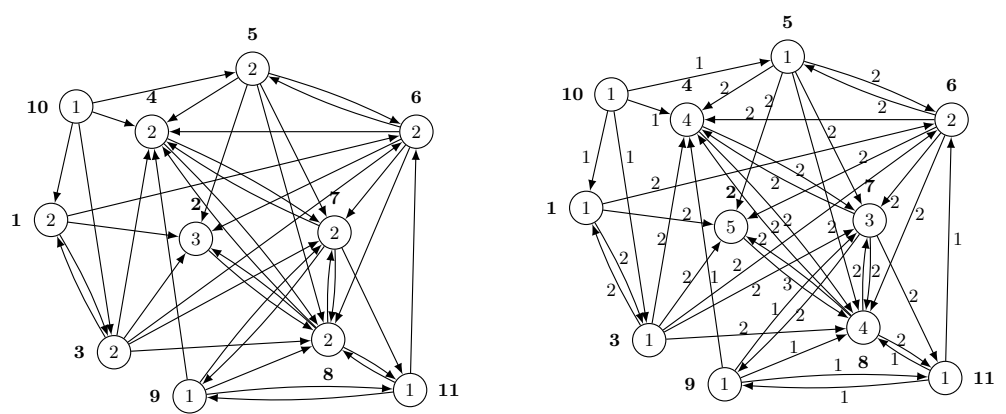

Fig. 4. Student Government discussion network and the asssociated influence graph.

\begin{tabular}{|c|c|c|c|c|c|c|c|c|}
\hline Node & $C_{D}^{-}$ & $C_{D}^{+}$ & $C_{C}$ & $C_{B}$ & $\mathrm{Bz}$ & $\mathrm{SS}$ & $C_{E}$ & $C_{S}$ \\
\hline 1 & 0.2 & 0.3 & $\mathbf{0 . 3 5 7}$ & 0.130 & $\mathbf{0 . 1 6 4}$ & $\mathbf{0 . 1 7 6}$ & $\mathbf{0 . 9 1}$ & $\mathbf{0 . 5 1 6}$ \\
2 & 0.5 & 0.1 & 0.200 & 0.195 & 0.154 & 0.076 & 0.45 & 0.515 \\
3 & 0.2 & $\mathbf{0 . 6}$ & $\mathbf{0 . 4 3 5}$ & 0.169 & $\mathbf{0 . 1 6 4}$ & $\mathbf{0 . 1 7 6}$ & $\mathbf{0 . 9 1}$ & $\mathbf{0 . 5 1 6}$ \\
4 & $\mathbf{0 . 7}$ & 0.2 & 0.208 & 0.204 & 0.005 & 0.009 & 0.55 & 0.500 \\
5 & 0.2 & $\mathbf{0 . 5}$ & 0.238 & 0.211 & $\mathbf{0 . 1 6 4}$ & $\mathbf{0 . 1 7 6}$ & $\mathbf{0 . 9 1}$ & $\mathbf{0 . 5 1 6}$ \\
6 & 0.4 & $\mathbf{0 . 5}$ & 0.238 & $\mathbf{0 . 3 0 4}$ & $\mathbf{0 . 1 6 4}$ & $\mathbf{0 . 1 7 6}$ & 0.82 & $\mathbf{0 . 5 1 6}$ \\
7 & $\mathbf{0 . 6}$ & $\mathbf{0 . 4}$ & 0.227 & $\mathbf{0 . 3 1 6}$ & 0.005 & 0.009 & 0.64 & 0.500 \\
8 & $\mathbf{0 . 8}$ & $\mathbf{0 . 4}$ & 0.227 & 0.262 & 0.005 & 0.009 & 0.55 & 0.500 \\
9 & 0.2 & $\mathbf{0 . 4}$ & 0.227 & 0.193 & 0.005 & 0.009 & 0.82 & 0.500 \\
10 & 0.0 & $\mathbf{0 . 4}$ & $\mathbf{0 . 5 5 6}$ & 0.111 & $\mathbf{0 . 1 6 4}$ & $\mathbf{0 . 1 7 6}$ & $\mathbf{0 . 9 1}$ & $\mathbf{0 . 5 1 6}$ \\
11 & 0.3 & 0.3 & 0.227 & $\mathbf{0 . 3 0 6}$ & 0.005 & 0.009 & 0.82 & 0.500 \\
\hline
\end{tabular}

Table 3. Comparison for the Student Government discussion networ and $q=6$.

distant nodes through paths. Nevertheless, nodes 1, 3 and 10, as well as ministers 5 and 6 , have a high score for measures Bz, SS and $C_{S}$. Thi is so since the spread of the influence over the other students, starting from the coalitions where they participate, is often necessary to overcome the required quota $q$. The same occurs for $C_{E}$, except for the minister 6 , which is a bit less central.

\section{Conclusions and future work}

Our main motivation in this work was to use influence games as a way to propose additional centrality measures coming from the field of cooperative game theory. The framework of influence games derives a connection between social network analysis and spread of influence in decision processes. We exploit this link with simple game theory to propose new centrality measures: Banzhaf, ShapleyShubik, Effort and Satisfaction. This is the first approach to apply power indices as centrality measures for social networks (for specific game-theoretic networks [9] has been used the Shapley-Shubik index as centrality measure). Our results do not contradict the relevance criteria provided by traditional centrality measures like degree centrality, closeness or betweenness. In some cases such 
measurements are similar to our measurements, but there are also cases where the results have been quite different. Indicating that an additional study on more realistic social networks is of interest.

Our proposal can be extended to other power indices [5] and measures, it will be of interest to determine which of them provide relevant rankings for social network analysis. Finally, we want to mention that there are other well known concepts related with players in simple games, such as dummy, vetoer or dictators [13], that could provide interesting properties of actors in a social network.

\section{References}

1. H. Aziz. Algorithmic and complexity aspects of simple coalitional games. PhD Thesis, Department of Computer Science, University of Warwick, 2009.

2. W. de Nooy, A. Mrvar, and V. Batagelj. Exploratory social network analysis with Pajek, volume 27 of Structural analysis in the social sciences. Cambridge Univ. Press, 2005.

3. M. G. Everett and S. P. Borgatti. The centrality of groups and classes. Journal of Mathematical Sociology, 23(3):181-201, 1999.

4. L. C. Freeman. Centrality in social networks: Conceptual clarification. Social Networks, 1(3):215-239, 1979.

5. J. Freixas. Power indices. In J. J. Cochran, L. A. Cox, P. Keskinocak, J. P. Kharoufeh, and J. C. Smith, editors, Wiley Encyclopedia of Operations Research and Management Science, volume 8. John Wiley \& Sons, 2011.

6. V. Hlebec. Recall versus recognition: Comparison of two alternative procedures for collecting social network data. In A. Ferligoj and T. Kramberger, editors, International Conference on Methodology and Statistics, 1992. Proceedings, pages 121-128, 1993.

7. D. Kempe, J. Kleinberg, and É. Tardos. Maximizing the spread of influence through a social network. In L. Getoor, T. E. Senator, P. Domingos, and C. Faloutsos, editors, Proceedings of the Ninth ACM SIGKDD International Conference on Knowledge Discovery and Data Mining, 2003, pages 137-146, 2003.

8. V. Latora and M. Marchiori. A measure of centrality based on network efficiency. New Journal of Physics, 9(6), 2007.

9. T. P. Michalak, K. V. Aadithya, P. L. Szczepański, B. Ravindran, and N. R. Jennings. Efficient computation of the Shapley value for game-theoretic network centrality. Journal of Artificial Intelligence Research, 46:607-650, 2013.

10. X. Molinero, F. Riquelme, and M. J. Serna. Social influence as a voting system: A complexity analysis of parameters and properties. CoRR, abs/1208.3751v3, 2014.

11. J. L. Moreno. The sociometry reader. Free Press, 1960.

12. J. Sun and J. Tang. A survey of models and algorithms for social influence analysis. In Aggarwal, editor, Social network data analytics, pages 177-214. 2011.

13. A. Taylor and W. Zwicker. Simple games: Desirability relations, trading, pseudoweightings. Princeton University Press, 1999.

14. R. van den Brink, A. Rusinowska, and F. Steffen. Measuring power and satisfaction in societies with opinion leaders: dictator and opinion leader properties. Homo Oeconomicus, 28(1-2):161-185, 2011.

15. S. Wasserman and K. Faust. Social network analysis: Methods and applications, volume 8 of Structural analysis in the social sciences. Cambridge Univ. Press, 1994. 